\title{
Primary adenocarcinoma of the renal pelvis, ureter and the urinary bladder: A case report and review of the literature
}

\author{
XING XIONG ${ }^{*}$, LINGHUA JIA ${ }^{*}$ and JINGEN WANG \\ Department of Urology, Jiangxi Provincial People's Hospital, Nanchang, Jiangxi 330006, P.R. China
}

Received January 10, 2015; Accepted December 7, 2015

DOI: $10.3892 / 01.2016 .4151$

\begin{abstract}
Primary adenocarcinoma is a rare type of urological neoplasm. The present study reports the case of a 55-year-old man with multifocal adenocarcinoma of the renal pelvis, ureter and urinary bladder that occurred in association with a large cystic calculus and perinephric abscess. The patient had suffered from gross hematuria for 2 years and right flank pain for 2 months. Following a series of investigations, a large cystic calculus with multiple tumors in the renal pelvis and ureter was identified. Multifocal tumors and a large calculus were located in the bladder using a cystoscope. The pathological report of 3 individual biopsies revealed a moderately differentiated tubular adenocarcinoma. Right nephrectomy, ureterectomy, radical cystectomy and left ureterocutaneostomy were performed. The pathological investigation revealed a moderately differentiated adenocarcinoma of the renal pelvis, ureter and urinary bladder. No additional treatment was administered and the patient remains alive at follow-up without disease recurrence or metastasis. Although uncommon, the development of a tumor is possible in patients that possess long-standing urolithiasis, particularly when accompanied by hydronephrosis or infection.
\end{abstract}

\section{Introduction}

The majority of malignant renal pelvic and ureteral tumors are of epithelial origin (1). Among these, $90 \%$ are transitional cell carcinoma (1). Transitional cell carcinoma cells grow in aggregates, whilst adenocarcinoma cells grow in tubules, with abundant cytoplasm and enlarged, hyperchromatic nuclei. However, pure adenocarcinomas of the urothelium are rare, accounting for $<1 \%$ of all urothelial

Correspondence to: Professor Jingen Wang, Department of Urology, Jiangxi Provincial People's Hospital, 92 Ai Guo Road, Nanchang, Jiangxi 330006, P.R. China

E-mail: dorxiong@163.com

*Contributed equally

Key words: adenocarcinoma, cystic calculus, renal pelvic tumor, ureter tumor, bladder tumor tumors; and for adenocarcinomas to be located in the pelvis is uncommon (2). Adenocarcinoma of the urinary bladder is also a rare malignancy that accounts for $<2 \%$ of the primary bladder cancers $(3,4)$. Common treatment may include nephrectomy, ureterectomy and partial cystectomy (5). The present study reports a case of multifocal primary adenocarcinoma of the renal pelvis, ureter and urinary bladder that occurred in association with a large cystic calculus and perinephric abscess.

\section{Case report}

A 55-year-old male nonsmoker presented with gross hematuria that had lasted for two years, right flank pain that had lasted for two months and no fever. At the time of admission, the patient's body temperature and blood pressure were $37.1^{\circ} \mathrm{C}$ and 107/69 mmHg, respectively. A general physical examination revealed pallor. The abdominal examination revealed a 20x $15 \mathrm{~cm}$ smooth, firm, non-tender and ballotable mass in the right lumbar area that extended to the right hypochondrium. Routine hematological investigations revealed a white blood cell count of $7.9 \times 10^{9}$ cells $/ 1$ (normal range, $3.5 \times 10^{9}-9.5 \times 10^{9}$ cells $/ 1$ ), a hemoglobinlevelof $89 \mathrm{~g} / 1$ (normalrange, $130-175 \mathrm{~g} / \mathrm{l}$ )andaplateletcount of $493 \times 10^{9}$ platelets/1(normal range, $100 \times 10^{9}-300 \times 10^{9}$ platelets $/ 1$ ). The urine examination revealed packed pus cells and red blood cells, and the culture was sterile. The plain abdominal radiograph revealed large cystic calculi. The computed tomography (CT) scan revealed a multifocal tumor in the right pelvis and ureter and a normally-functioning left kidney (Fig. 1). Technetium-99m diethylene-triamine-pentaacetic acid renal scintigraphy revealed a non-functioning right kidney. The cystoscope revealed large calculi in the bladder and multifocal tumors along the wall of the urinary bladder. The pathological report of 3 tissue samples revealed a moderately differentiated tubular adenocarcinoma.

The diagnosis was multifocal carcinoma of the renal pelvis, ureter and urinary bladder, which occurred in association with a large cystic calculus and perinephric abscess. Right nephrectomy, ureterectomy, radical cystectomy and left ureterocutaneostomy were performed. Intraoperatively, the right kidney was markedly enlarged, possessed a perinephric adhesion and abscess and contained $\sim 1,500 \mathrm{ml}$ of urine in the renal pelvis. The right ureter was dilated, markedly enlarged and contained a perinephric adhesion. A gross pathological examination revealed that the kidney and attached ureter 


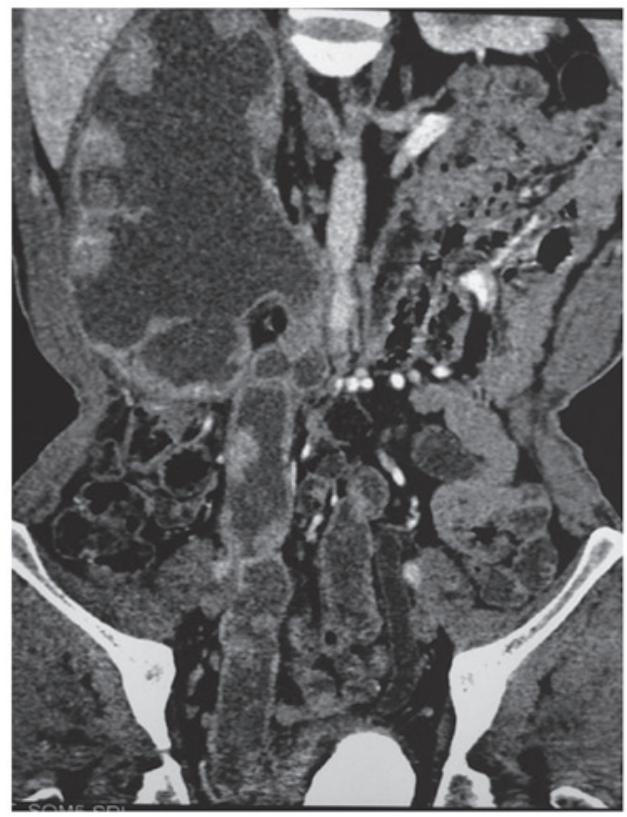

Figure 1. Computed tomography revealed a multifocal tumor in the right pelvis and ureter and a normally functioning left kidney.

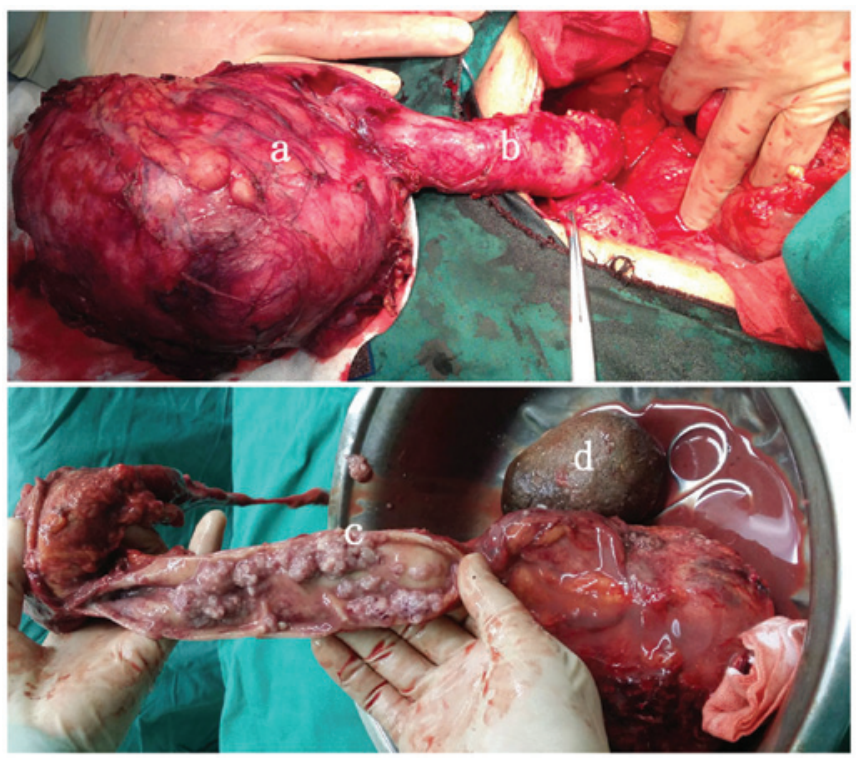

Figure 2. (a) The right kidney. (b) The right ureter. (c) Multifocal tumors in the ureter. (d) The large cystic calculus.

measured $13 \times 8 \times 6 \mathrm{~cm}$ and $17 \times 3 \mathrm{~cm}$, respectively. The bladder measured $8 \times 7 \times 3 \mathrm{~cm}$ and the attached prostate was $3 \times 3 \times 1 \mathrm{~cm}$. The cut section revealed a dilated calyx, which exhibited the loss of the corticomedullary junction, and multifocal tumors in the renal pelvis, ureter and urinary bladder that were associated with a large cystic calculus (Fig. 2). Microscopic examinations revealed the characteristics of adenocarcinoma. It was observed that the cells were growing in tubules, and exhibited abundant cytoplasm and enlarged, hyperchromatic nuclei (Fig. 3). Lymphovascular and perinephric fat invasions were not observed. No additional treatment was administered and the patient remains alive at follow-up with no tumor recurrence or metastasis.

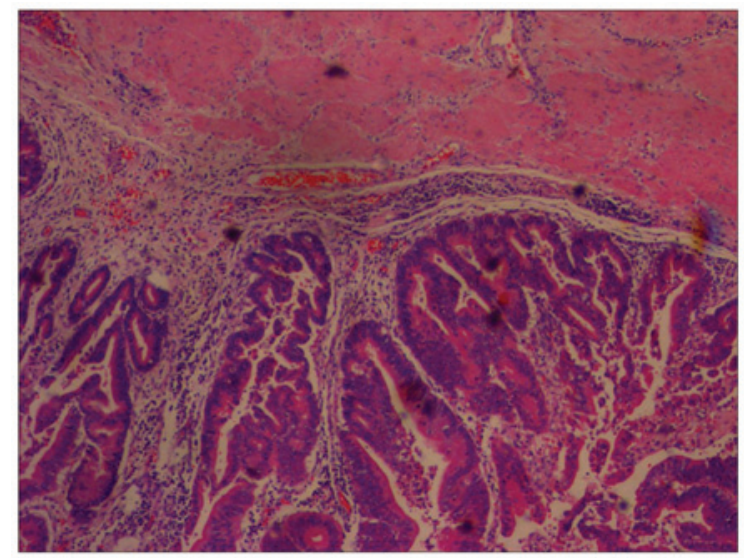

Figure 3. Microscopic examination revealed the characteristics of adenocarcinoma. The cells were growing in tubules, exhibiting abundant cytoplasm and enlarged, hyperchromatic nuclei. Staining, hematoxylin and eosin; magnification, $\mathrm{x} 100$.

\section{Discussion}

The majority of tumors of the renal pelvis and ureter have a distinct pathology (1). The most frequently observed tumor is urothelial carcinoma, which accounts for $90 \%$ of all renal pelvis and ureter tumors (1). The other tumors observed tend to be squamous cell carcinoma, adenocarcinoma or leiomyosarcoma (1). Among the special types of tumors, primary adenocarcinomas are relatively uncommon, and account for $1 \%$ of all renal, pelvis and ureter tumors (2). Only a small number of individual cases are reported in the literature. Renal pelvic and ureter adenocarcinomas are often associated with chronic inflammation and subsequent chronic infection, hydronephrosis and urinary calculi $(6,7)$. In 1946, Ackerman reported the first case of mucinous adenocarcinoma of the pelvis (8). At present, $\sim 100$ cases have been reported in the English medical literature (PubMed, Elsevier, Medline, Springer and EBSCO). The highest numbers of reported cases are from Japan (12 cases) and India (10 cases), while other areas of the world have reported occasional cases (9).

The pathogenesis of the present renal pelvis and ureter tumors is not clear. The suggested pathogenesis considers the frequent association with chronic irritation, infection, inflammation, hydronephrosis and urinary calculi. Glandular metaplasia of the urothelium may develop as a response to injury, and can often progress to dysplasia and adenocarcinoma (10). The majority of cases are reported in patients older than 60 years, and there is no evidence to suggest that the occurrence of the tumor is associated with gender $(5,11)$. Hematuria is the most common symptom at presentation, while loin pain and a palpable abdominal mass signifies a late stage in the disease; however, patients may be asymptomatic (12). Over two thirds of cases are associated with urolithiasis and hydronephrosis (12).

In addition to urothelial adenocarcinoma, adenocarcinoma of the urinary bladder is a rare malignancy that accounts for $<2 \%$ of primary bladder cancers $(13,14)$. Adenocarcinoma may be classified into three groups, consisting of the primary vesicle, urachal and metastatic adenocarcinomas (12). Metastatic adenocarcinoma is one of the most common forms of 
adenocarcinoma (15). The diagnosis, treatment and prognosis of metastatic adenocarcinoma are closely associated with the primary site, which may include the rectum, stomach, endometrium, breast, prostate or ovary (6). Adenocarcinoma of the bladder is considered to be associated with a poor prognosis in the majority of studies (13). However, previous observations indicate that bladder adenocarcinoma may have the same natural history as urothelial adenocarcinoma. An analysis of survival rate from the Netherlands Cancer Registry indicated that the survival of patients with muscle-invasive bladder adenocarcinoma is similar to the survival of patients with muscle-invasive urothelial carcinoma (16). Ghoneim et al (17) also identified that there are no statistical differences between bladder and urothelial carcinomas in terms of mortality.

Adenocarcinomas of the urinary bladder may be subclassified into urachal (U) and non-urachal (NU) adenocarcinomas (14). Zhang et al (14) reviewed the data of 21 cases with primary adenocarcinoma of the bladder over a 10 -year period, in order to investigate the variation between urachal and non-urachal adenocarcinomas in terms of patient survival. Numerous studies agree that urachal and non-urachal tumors probably exist together in the majority of cases $(14,18)$. Although the two tumors possess the same pathogenesis, there appears to be clinical differences between the two types. At present, it remains unclear whether the survival of patients with urachal adenocarcinoma varies from that of patients with non-urachal adenocarcinomas. Wright et al (19) identified that urachal cancer possessed a significantly better prognosis than non-urachal cancer, while Mostofi et al (20) hypothesized that urachal adenocarcinomas were associated with a significantly worse prognosis. In addition, Dandekar et al (21) considered there to be no significant differences between the two carcinomas. Zhang et al (14) identified that urachal adenocarcinoma was associated with a more favorable outcome. However, the variation between the two types was not statistically significant using the Kaplan-Meier estimates $(\mathrm{P}=0.0763)$.

Primary adenocarcinoma of the urological region requires differentiation from more common metastatic adenocarcinomas, including direct, lymphatic and hematogenous spread $(22,23)$. The principal primary organs to be considered include the prostate, colon, female genital tract, appendix, stomach and breast $(22,23)$. The presence of a flat carcinoma in situ provides evidence to support the theory that a primary vesical adenocarcinoma may be considered a separate entity $(24,25)$. Flat carcinomas in situ are challenging to document, particularly in transurethral resection specimens due to extensive thermal artifacts that may be caused by complete sampling, and the presence of mucosal ulcerations $(24,25)$. Also, secondary adenocarcinoma tends to colonize the native epithelium, which may mimic a carcinoma in situ component $(24,25)$. The most frequent and challenging differential diagnosis remains to be metastatic or directly spreading colonic adenocarcinoma. The latter is virtually indistinguishable based on histomorphology and immunohistochemistry $(24,25)$. Therefore, differential diagnosis is even more challenging on small biopsies that possess poorly differentiated tumors.

Lauritzen et al (26) and Sidharth et al (27) reported cases of patients that underwent a radical nephrectomy and total ureterectomy, including an ipsilateral bladder cuff excision, to treat a primary carcinoma of the upper urinary tract. The patient in the present study was possessed multifocal adenocarcinoma of the renal pelvis, ureter and the urinary bladder that occurred in association with a large cystic calculi and perinephric abscess. To the best of our knowledge, the present study is the first to report a case of this type. Right nephrectomy, ureterectomy, radical cystectomy and left ureterocutaneostomy were performed. The patient has now lived without a tumor for 6 months.

\section{References}

1. Spires SE, Banks ER, Cibull ML, Munch L, Delworth M and Alexander NJ: Adenocarcinoma of renal pelvis. Arch Pathol Lab Med 117: 1156-1160, 1993.

2. Sagnotta A, Dente M, Socciarelli F, Cacchi C, Stoppacciaro A and Balducci G: Primary adenocarcinoma of the renal pelvis: Histologic features of a stepwise process from intestinal hyperplasia to dysplasia in a patient with chronic renal abscess. Int J Surg Pathol 22: 182-185, 2014.

3. Roy S, Smith MA, Cieply KM, Acquafondata MB and Parwani AV: Primary bladder adenocarcinoma versus metastatic colorectal adenocarcinoma: A persisting diagnostic challenge. Diagn Pathol 7: 151,2012.

4. Nielsen K and Nielsen KK: Adenocarcinoma in exstrophy of the bladder - the last case in Scandinavia? A case report and review of literature. J Urol 130: 1180-1182, 1983.

5. Tyler A: Urothelial cancers: Ureter, renal pelvis, and bladder. Semin Oncol Nurs 28: 154-162, 2012.

6. Ueda T, Okumi M, Ichimaru N, Itoh K, Matsuoka Y and Fujimoto N: Mucinous adenocarcinoma of the renal pelvis in the horseshoe kidney: A case report. Hinyokika Kiyo 48: 187-189, 2002 (In Japanese).

7. Hudson J, Arnason T, Merrimen JL and Lawen J: Intestinal type villous adenoma of the renal pelvis. Can Urol Assoc J 7: E138-E142, 2013.

8. Ackerman LV: Mucinous adenocarcinoma of the pelvis of the kidney. J Urol 55: 36, 1946.

9. Punia RP, Mundi I, Arora K, Dalal A and Mohan H: Primary adenocarcinoma of ureter mimicking pyelonephritis. Urol Ann 2: 42-43, 2010.

10. Yonekawa M, Hoshida Y, Hanai J, Tuboniwa N, Katayama K, Kishimoto T and Aozasa K: Catheterized urine cytology of mucinous carcinoma arising in the renal pelvis. A case report. Acta Cytol 44: 442-444, 2000.

11. Xambre L, Cerqueira M, Cardoso A, Correia T, Macedo Dias A, Carreira $\mathrm{F}$ and Galán T: Primary mucinous adenocarcinoma of the renal pelvis - adicional case report]. Actas Urol Esp 33: 200-204, 2009.

12. Abbas M, Kramer MW, Spieker T, Herrman TR, Merseburger AS, Müller KM, Kuczyk MA, Becker JU and Kreipe HH: Primary mucinous adenocarcinoma of the renal pelvis with carcinoma in situ in the ureter. J Egypt Natl Canc Inst 26: 51-54, 2014.

13. Zhong M, Gersbach E, Rohan SM and Yang XJ: Primary adenocarcinoma of the urinary bladder: Differential diagnosis and clinical relevance. Arch Pathol Lab Med 137: 371-381, 2013.

14. Zhang H, Jiang H, Wu Z, Fang Z, Fan J and Ding Q: Primary adenocarcinoma of the urinary bladder: A single site analysis of 21 cases. Int Urol Nephrol 45: 107-111, 2013.

15. Roy S and Parwani AV: Adenocarcinoma of the urinary bladder. Arch Pathol Lab Med 135: 1601-1605, 2011.

16. Ploeg M, Aben KK, Hulsbergen-van de Kaa CA, Schoenberg MP, Witjes JA and Kiemeney LA: Clinical epidemiology of nonurothelial bladder cancer: Analysis of the Netherlands Cancer Registry. J Urol 183: 915-920, 2010.

17. Ghoneim MA, Abdel-Latif M, el-Mekresh M, Abol-Enein H, Mosbah A, Ashamallah A and el-Baz MA: Radical cystectomy for carcinoma of the bladder: 2,720 consecutive cases 5 years later. J Urol 180: 121-127, 2008.

18. Cho SY, Moon KC, Park JH, Kwak C, Kim HH and Ku JH: Outcomes of Korean patients with clinically localized urachal or non-urachal adenocarcinoma of the bladder. Urol Oncol 31: 24-31, 2013.

19. Wright JL, Porter MP, Li CI, Lange PH and Lin DW: Differences in survival among patients with urachal and nonurachal adenocarcinomas of the bladder. Cancer 107: 721-728, 2006. 
20. Mostofi FK, Thomson RV and Dean AL Jr: Mucous adenocarcinoma of the urinary bladder. Cancer 8: 741-758, 1955.

21. Dandekar NP, Dalal AV, Tongaonkar HB and Kamat MR: Adenocarcinoma of bladder. Eur J Surg Oncol 23: 157-160, 1997.

22. Wang HL, Lu DW, Yerian LM, Alsikafi N, Steinberg G, Hart J and Yang XJ: Immunohistochemical distinction between primary adenocarcinoma of the bladder and secondary colorectal adenocarcinoma. Am J Surg Pathol 25: 1380-1387, 2001.

23. Torenbeek R, Lagendijk JH, Van Diest PJ, Bril H, van de Molengraft FJ and Meijer CJ: Value of a panel of antibodies to identify the primary origin of adenocarcinomas presenting as bladder carcinoma. Histopathology 32: 20-27, 1998.
24. Suh N, Yang XJ, Tretiakova MS, Humphrey PA and Wang HL: Value of CDX2, villin, and alpha-methylacyl coenzyme A racemase immunostains in the distinction between primary adenocarcinoma of the bladder and secondary colorectal adenocarcinoma. Mod Pathol 18: 1217-1222, 2005.

25. Raspollini MR, Nesi G, Baroni G, Girardi LR and Taddei GL: Immunohistochemistry in the differential diagnosis between primary and secondary intestinal adenocarcinoma of the urinary bladder. Appl Immunohistochem Mol Morphol 13: 358-362, 2005.

26. Lauritzen AF, Kvist E, Bredesen J and Luke M: Primary carcinoma of the upper urinary tract. Acta Pathol Microbiol Immunol Scand A 95: 7-10, 1987.

27. Sidharth, Maskey P, Chalise PR, Shrestha A, Sharma UK, Gyawali PR, Shrestha GK and Joshi BR: Primary mucinous adenocarcinoma of the renal pelvis and ureter. Nepal Med Coll J 13: 229-230, 2011. 